Check for updates

Cite this: Chem. Sci., 2019, 10, 11041

๑ All publication charges for this article have been paid for by the Royal Society of Chemistry

Received 29th June 2019

Accepted 30th September 2019

DOI: $10.1039 / c 9 s c 03219 c$

rsc.li/chemical-science

\section{A pencil-and-paper method for elucidating halide double perovskite band structures $\uparrow$}

\author{
Adam H. Slavney, (D) a Bridget A. Connor, (D) ${ }^{a}$ Linn Leppert ${ }^{\mathrm{b}}$ \\ and Hemamala I. Karunadasa (ID *ac
}

\begin{abstract}
Halide double perovskites are an important emerging alternative to lead-halide perovskites in a variety of optoelectronic applications. Compared to $A B X_{3}$ single perovskites ( $A=$ monovalent cation, $X=$ halide), $\mathrm{A}_{2} \mathrm{BB}^{\prime} \mathrm{X}_{6}$ double perovskites exhibit a wider array of compositions and electronic structures, promising finer control over physical and electronic properties through synthetic design. However, a clear understanding of how chemical composition dictates the electronic structures of this large family of materials is still lacking. Herein, we develop a qualitative Linear Combination of Atomic Orbitals (LCAO) model that describes the full range of band structures for double perovskites. Our simple model allows for a direct connection between the inherently local bonding between atoms in the double perovskite and the resulting delocalized bands of the solid. In particular, we show how bands in halide double perovskites originate from the molecular orbitals of metal-hexahalide coordination complexes and describe how these molecular orbitals vary within a band. Our results provide both an enhanced understanding of known perovskite compositions and predictive power for identifying new compositions with targeted properties. We present a table, which permits the position of the conduction band minimum and valence band maximum in most double perovskites to be immediately determined from the frontier atomic orbitals of the B-site metals. Using purely qualitative arguments based on orbital symmetries and their relative energies, the direct/indirect nature of the bandgap of almost all halide double perovskites can thus be correctly predicted. We hope that this theory provides an intuitive understanding of halide double perovskite band structures and enables lessons from molecular chemistry to be applied to these extended solids.
\end{abstract}

\section{Introduction}

Perovskites encompass a vast structural and compositional diversity featuring 0-, 1-, 2-, and 3-dimensional lattices incorporating s-, p-, d-, and f-block metals. This diversity is reflected in the widely varying properties of perovskites, which have been studied as superconductors, ${ }^{\mathbf{1}}$ catalysts, ${ }^{2}$ rare-earth phosphor hosts, ${ }^{3}$ and conductors for lithium ions, ${ }^{4}$ oxide ions, ${ }^{5}$ and protons. ${ }^{6}$ The most-studied perovskites are oxides. However, halide perovskites have recently attracted a resurgence of interest. This is largely due to the outstanding photovoltaic performance of the lead-halide 3D perovskites (Fig. 1A), which have afforded solar cells with power conversion efficiencies exceeding $24 \%{ }^{7}$ only 10 years since their initial demonstration. ${ }^{8}$

${ }^{a}$ Department of Chemistry, Stanford University, Stanford, CA 94305, USA. E-mail: hemamala@stanford.edu

${ }^{b}$ Institute of Physics, University of Bayreuth, Bayreuth, 95440, Germany

'Stanford Institute for Materials and Energy Sciences, SLAC National Accelerator Laboratory, Menlo Park, California 94025, USA

$\dagger$ Electronic supplementary information (ESI) available: Computation methods, supplementary figures, detailed group theoretical analysis, and analysis of $\pi$-bonding bands. See DOI: 10.1039/c9sc03219c
However, the inherent instability, high toxicity, and environmental mobility of these water-soluble $\mathrm{Pb}^{2+}$ salts ${ }^{\mathbf{9}, 10}$ have instigated a large effort to identify other compositions that possess similar electronic properties.

The recent revival of interest in halide double perovskites (elpasolites), ${ }^{\mathbf{1 1 - 1 3}}$ which have been known since the $1880 \mathrm{~s},{ }^{\mathbf{1 4}}$ has been primarily motivated by the desire to replicate the electronic structures of the lead-halide perovskites ${ }^{\mathbf{1 5}}$ in alternative compositions. We have been deeply invested in this effort, primarily through the introduction of double perovskites as potential solar absorbers ${ }^{\mathbf{1 1}}$ and the synthesis of targeted new compositions for obtaining suitable bandgaps for sunlight absorption. ${ }^{16,17}$ The $\mathrm{A}_{2}^{\mathrm{I}} \mathrm{BB}^{\prime} \mathrm{X}_{6}$ double perovskites $(\mathrm{A}=$ monovalent cation, $\mathrm{X}=$ halide; Fig. $1 \mathrm{~B}$ ) have the same connectivity as the $\mathrm{AB}^{\mathrm{II}} \mathrm{X}_{3}$ single perovskites but divide the formally 2+ charge at the octahedral site (the B site) unevenly over two sites, allowing for the incorporation of metals with oxidation states from +1 to +4 . This uneven division is advantageous because almost every element has a stable oxidation state between +1 and +4 allowing a wider variety of metals to be incorporated. The compositional flexibility of double perovskites leads to an incredible degree of complexity and diversity in their electronic structures. In these 

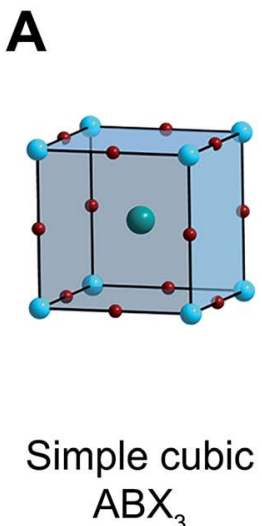
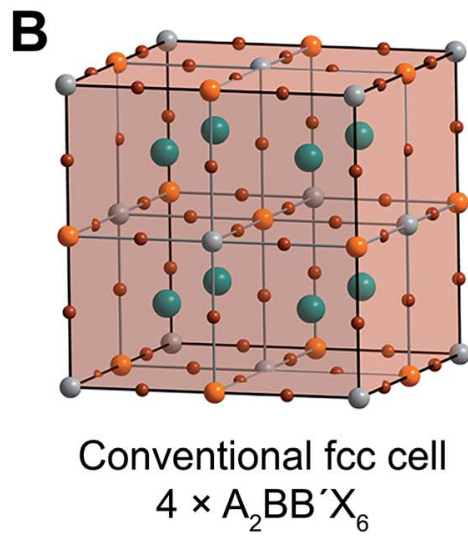

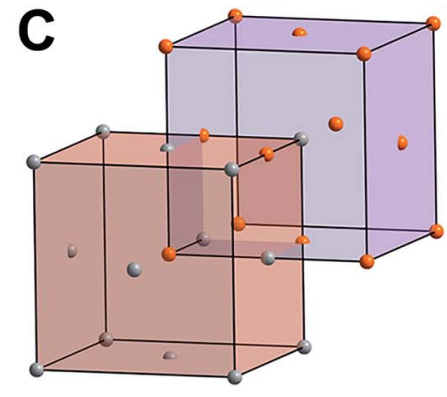

Interpenetrating B/B' fcc lattices
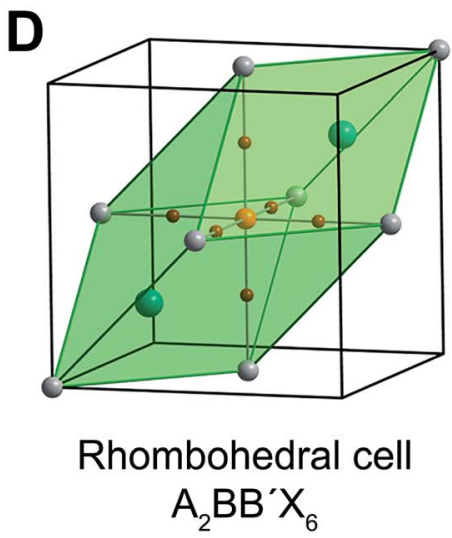

Fig. 1 Ball-and-stick representations of $(A)$ the unit cell of the cubic single perovskite $\mathrm{CsPb}^{\prime \prime} \mathrm{Br}_{3}$ and (B) the conventional (four formula units) face-centered-cubic ( $f c c$ ) cell of the double perovskite $\mathrm{Cs}_{2} \mathrm{Ag}^{\prime} \mathrm{Bi}^{\prime \prime \prime} \mathrm{Br}_{6}$. (C) Depiction of the interpenetrating $\mathrm{B} / \mathrm{B}^{\prime}$ fcc lattices present in rock-saltordered double perovskites such as $\mathrm{Cs}_{2} \mathrm{AgBiBr}_{6}$. (D) The rhombohedral (one formula unit) unit cell of $\mathrm{Cs}_{2} \mathrm{AgBiBr}_{6}$. Turquoise, orange, silver, green, and brown spheres represent $\mathrm{Pb}, \mathrm{Bi}, \mathrm{Ag}, \mathrm{Cs}$, and $\mathrm{Br}$ atoms, respectively.

materials, a single metal substitution can drastically modify the band edges, leading to the observation of direct-allowed, directforbidden, and indirect bandgaps, both large and small in magnitude, all within the same structural family. ${ }^{11-13,17-21}$ It is increasingly evident that double perovskites afford a highly tunable electronic platform; however, in order to realize their true potential, we must first understand the origins of their electronic diversity.

With computing time now quite cheap, electronic structures are determined almost exclusively through density functional theory (DFT) calculations. Many such studies have been performed for halide perovskites, providing important insights to their optoelectronic properties..$^{12,13,17-26}$ These methods and the band diagrams they produce, however, are specific to the perovskite under study and not necessarily generalizable. Additionally, typical band structure diagrams often obscure the local chemical information contained in the electronic structure. For instance, the atomic composition of the bands, the directionality ( $\sigma$ and $\pi$ character) and symmetry (bonding, antibonding, nonbonding nature) of the interactions between the orbitals that compose the bands, and the variation of these parameters within a band, are often not readily available from the final band structure.

In this contribution, we develop a simple theory, complementary to the existing DFT paradigm, which bridges our chemical understanding of metal-halide coordination complexes and halide perovskite band structure. In particular, we flesh out the perovskite band structure with atomistic detail, emphasizing the local molecular interactions that ultimately dictate the bandgap and band dispersion. We construct the band diagrams using arguments founded on the symmetries and relative energies of atomic orbitals-methods that are intuitive to both molecular and solid-state chemists and that have a long history of correctly predicting material properties from simple starting points.

We will show that a linear combination of atomic orbitals (LCAO) approach, ${ }^{27,28}$ in particular the qualitative analysis demonstrated by Hoffmann and others, ${ }^{29}$ accurately reproduces the electronic band structure of halide double perovskites near their high-symmetry $\mathbf{k}$ points. For the lead-halide perovskites, a similar qualitative approach was recently reported, ${ }^{30}$ and a handful of quantitative (but closely related) tight-binding models have been developed. ${ }^{31,32}$ Additionally, tight-binding models for some oxide perovskites are also available. ${ }^{33-35}$ However, to our knowledge, this is the first report of LCAO analysis on halide double perovskites. We hope that this work will develop a general, intuitive picture of double perovskite electronic structure, provide a simple check for theoretical investigations of electronic structure, and serve as a useful starting point for exploratory syntheses of new double perovskites.

The paper is organized as follows: in Section 2, we present our LCAO method for constructing extended electronic states, which is a modification of prior approaches (Fig. 2). ${ }^{29,30}$ In Section 3, we apply the LCAO method to two case studies demonstrating its utility for understanding the electronic structure of known halide double perovskites. We also derive general rules for predicting band extrema in halide double perovskites. In Section 4, we condense our results to a simple table (Table 1), where knowledge (based on measurement or chemical intuition) of the frontier atomic orbitals of a given perovskite composition enables immediate determination of the conduction band minimum (CBM) and valence band maximum (VBM) for the vast majority of double perovskites. We compare our predictions against DFT results for many known halide double perovskites and show overwhelming agreement. We conclude by discussing the limitations and possible extensions of the method.

\section{Methods}

For a general overview of the LCAO method, we refer the reader to one of several excellent texts; $;^{29,36,37}$ a brief primer is also provided in the ESI. $\dagger$ Our approach deviates from prior LCAO schemes, which typically apply translational symmetry to the molecular metal-ligand complex in order to build the extended solid..$^{29,30}$ In order to generalize to all halide double perovskites 


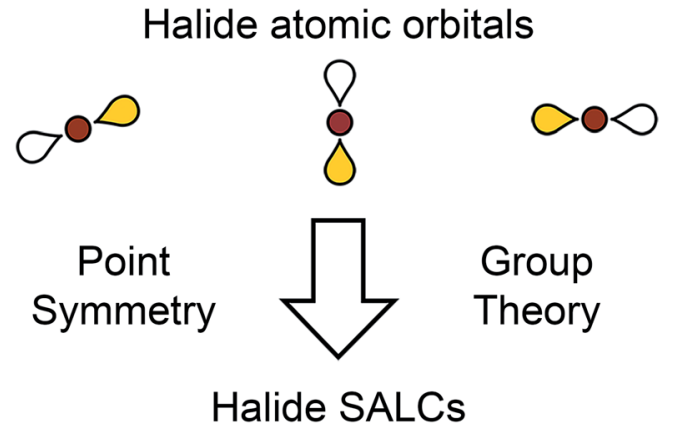

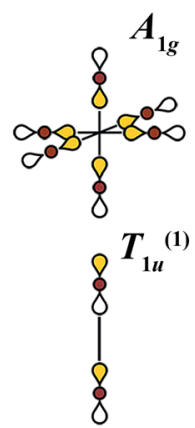
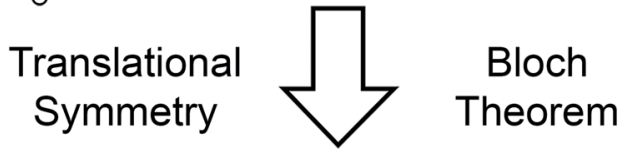

Halide Bloch waves
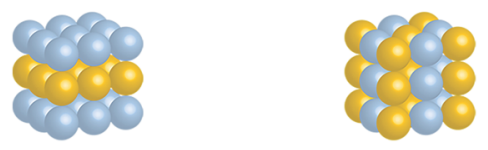

\section{Add Metal Orbitals}

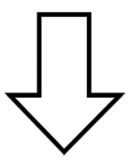

\section{Evaluate
Bonding}

\section{Band structure}

Fig. 2 Schematic showing the steps of the Linear Combination of Atomic Orbitals (LCAO) method. Halide p orbitals are used to form symmetry adapted linear combinations (SALCS), which are propagated through three-dimensions to form halide-only Bloch waves. Metal orbitals are then introduced to evaluate band dispersion. Only $\sigma$ bonds are considered here. See ESI $\uparrow$ for consideration of $\pi$ bonds.

(with any combination of B-site metals), here we apply translational symmetry to just the octahedrally arranged ligands and introduce metal-ligand interactions only after the extended electronic states have been constructed (Fig. 2).

The DFT calculations used to check our results were performed using the PBEsol exchange-correlation approximation as implemented in the VASP program code, including spinorbit coupling (SOC) self-consistently (see ESI $\uparrow$ for computational details).

\subsection{The double perovskite crystal structure and unit cell}

The $\mathrm{ABX}_{3}$ perovskite structure (Fig. 1A) consists of a 3D lattice of corner-sharing halide octahedra, with the (typically divalent) Bsite metal occupying the center of the octahedra. The (typically monovalent) A-site cation occupies the cuboctahedral cavities defined by eight corner-sharing metal-halide octahedra. The Asite cation (e.g. $\mathrm{Cs}^{+}, \mathrm{CH}_{3} \mathrm{NH}_{3}^{+}$) generally does not interact electronically with the $\mathrm{B}-\mathrm{X}$ sublattice and does not contribute meaningfully to the electronic structure near the band edges. Consequently, we will neglect it going forward.

Double perovskites contain two crystallographically distinct B sites. Most commonly, two distinct metal cations (differing in identity or oxidation state) occupy the B sites, alternating in all three directions (Fig. 1B). This ordering generates two interpenetrating face-centered-cubic (fcc) sublattices that are shifted by half a unit cell (in all three directions) from one another, similar to the $\mathrm{NaCl}$ rock-salt structure (Fig. 1C). Therefore, while cubic $\mathrm{ABX}_{3}$ perovskites have a primitive cubic lattice, the broken B-site symmetry gives $\mathrm{A}_{2} \mathrm{BB}^{\prime} \mathrm{X}_{6}$ double perovskites a facecentered-cubic (fcc) lattice, placing them in the $F m \overline{3} m$ space group. The conventional double perovskite fcc cell contains four formula units and, thus, is actually a supercell of the double perovskite lattice. The unit cell containing a single formula unit is a rhombohedral cell, which is the primitive unit cell of one of the fcc lattices (Fig. 1D). Unlike the cubic conventional cell, which has $90^{\circ}$ angles between its axes, the angle between the rhombohedral cell axes $\left(a_{1}, a_{2}\right.$, and $\left.a_{3}\right)$ is $60^{\circ}$. Geometric transformations between primitive and conventional fcc cells are provided in Fig. $\mathbf{S} 2 . \dagger$

\subsection{Constructing symmetry-adapted linear combinations (SALCs) of halide orbitals}

As the first step in the LCAO theory, we will consider only the halides and use group theory to find their symmetry-adapted linear combinations (SALCs). Here, we consider the 6 halides arranged in an octahedral geometry around the B site in the rhombohedral unit cell (Fig. 1D). Each halide contributes three orthogonal p orbitals: one $\sigma$-bonding orbital and two $\pi$-bonding orbitals. We will initially neglect the two $\pi$-bonding $\mathrm{p}$ orbitals. When only main group or $\mathrm{d}^{10}$ cations are in the $\mathrm{B}$ site, this simplification is valid as the band edges will consist of only $\sigma$ bonding states (see Fig. S1†). When a d $\mathrm{d}^{0}-\mathrm{d}^{9}$ or a lanthanide cation is present at one or both B sites, the $\pi$ interactions must be considered (shown in the ESI $\dagger$ ). Due to the rock-salt ordering of the $\mathrm{B}$ and $\mathrm{B}^{\prime}$ cations, the rhombohedral double-perovskite unit cell has $D_{3 d}$ point symmetry. However, because we initially neglect the metals and only consider the halides, we can treat the point symmetry as $O_{h}$ (and account for $D_{3 d}$ symmetry when applying translational symmetry). Using group theory, we generate the symmetry adapted linear combinations (SALCs) of the 6 halide orbitals: $A_{1 g}, E_{g}^{(1),(2)}$, and $T_{1 u}^{(1),(2),(3)}$ (Fig. 2, see Fig. $\mathrm{S} 4 \uparrow$ for full analysis) as expected for a molecular octahedral complex with $\sigma$ bonding.

\subsection{Applying translational symmetry}

To move from a molecular picture to that of an extended solid, we must propagate our halide SALCs using the translational symmetry of the double perovskite lattice. Translational symmetry is described mathematically by the Bloch theorem: 


$$
\psi(r+\mathbf{R})=\mathrm{e}^{\mathrm{i} \mathbf{k} \cdot \mathbf{R}} \psi(r)
$$

where $\psi(r)$ represents the wavefunction of a single unit cell (here a halide SALC) at the origin, the vector $\mathbf{R}$ describes the position of a second unit cell with respect to the origin, and the vector $\mathbf{k}$ describes how the phase of the wavefunction (the SALC) changes upon translation. Thus, the Bloch theorem states that the wavefunction in a crystalline solid may only change by a phase factor (the exponential factor) upon translating from one unit cell to the next (Fig. 3). The degree of that phase change is described by the translational quantum number $\mathbf{k}$. For three dimensional materials, $\mathbf{k}$ is a vector with coordinates derived from the three lattice vectors of the unit cell. We will focus our analysis on the high-symmetry $\mathbf{k}$ points, where the coordinates of $\mathbf{k}$ adopt values of either 0 or $\pi / a$ ( $a=$ unit-cell length). A coordinate of 0 denotes symmetric translation of the wavefunction along the corresponding direction $\left(\psi(r) \mathrm{e}^{\mathrm{i} \cdot 0 \cdot a}=\psi(r)\right)$ whereas a coordinate of $\pi / a$ denotes antisymmetric translation $\left(\psi(r) \mathrm{e}^{\mathrm{i} \cdot(\pi / a) \cdot a}=-\psi(r)\right)$. At lower-symmetry $\mathbf{k}$ points, $\mathbf{k}$ holds an intermediate value, resulting in some unit cells having wavefunctions with less than the maximal amplitude. For example, a coordinate of $\pi / 2 a$ results in every unit cell being out of phase with its second-nearest neighbours and in phase with its fourthnearest neighbours, while the first- and third-nearest neighbours are nodes with zero amplitude. The Bloch theorem thus produces periodic patterns of in-phase and/or out-of-phase SALCs, termed Bloch waves. High-symmetry $\mathbf{k}$ points produce Bloch waves where the wavefunction of every unit cell has maximal amplitude, allowing for strong bonding and antibonding interactions. Thus, band extrema (the most bonding or antibonding states within a band) typically occur at highsymmetry $\mathbf{k}$ points.

For our analysis, we will consider the three highest-symmetry k points of the fcc lattice: $\Gamma:(0,0,0), \mathbf{L}:(\pi / a, \pi / a, \pi / a)$, and $\mathbf{X}:(\pi /$ $a, 0, \pi / a)$. Here, $\boldsymbol{\Gamma}$ corresponds to in-phase SALCs along each axis, $\mathbf{L}$ to out-of-phase SALCs along each axis, and $\mathbf{X}$ to out-ofphase SALCs along the $a_{1}$ and $a_{3}$ axes and in-phase SALCs along the $a_{2}$ axis (see Fig. 3). These axes are defined with respect to the rhombohedral unit cell and point at $60^{\circ}$ angles with respect to one another. Visualizing translations along these non-orthogonal rhombohedral lattice vectors is rather difficult, so we use a geometric trick to transform the three rhombohedral vectors into two orthogonal vectors that lie within a Cartesian plane (see Fig. S2†).

We now apply translational symmetry to the SALCs to construct halide-based Bloch waves (Fig. 4). We arbitrarily pick one unit cell as the origin and place a halide SALC around the B (or $\mathrm{B}^{\prime}$ ) site. Note that for ease of visualization, the unit-cell origin in Fig. 4 has been shifted from that in Fig. 1D. We then translate the SALC along one of the two orthogonal vectors to the adjacent unit cell. Depending on the coordinate of $\mathbf{k}$ for our chosen direction, the phases of the orbitals in the new unit cell will either be in-phase or out-of-phase with respect to the SALC at the origin. We continue to propagate the Bloch wave by translating to additional unit cells, making use of both orthogonal vectors. This yields a Bloch wave propagated along two dimensions ( $x$ and $z$ in Fig. 4). Different combinations of

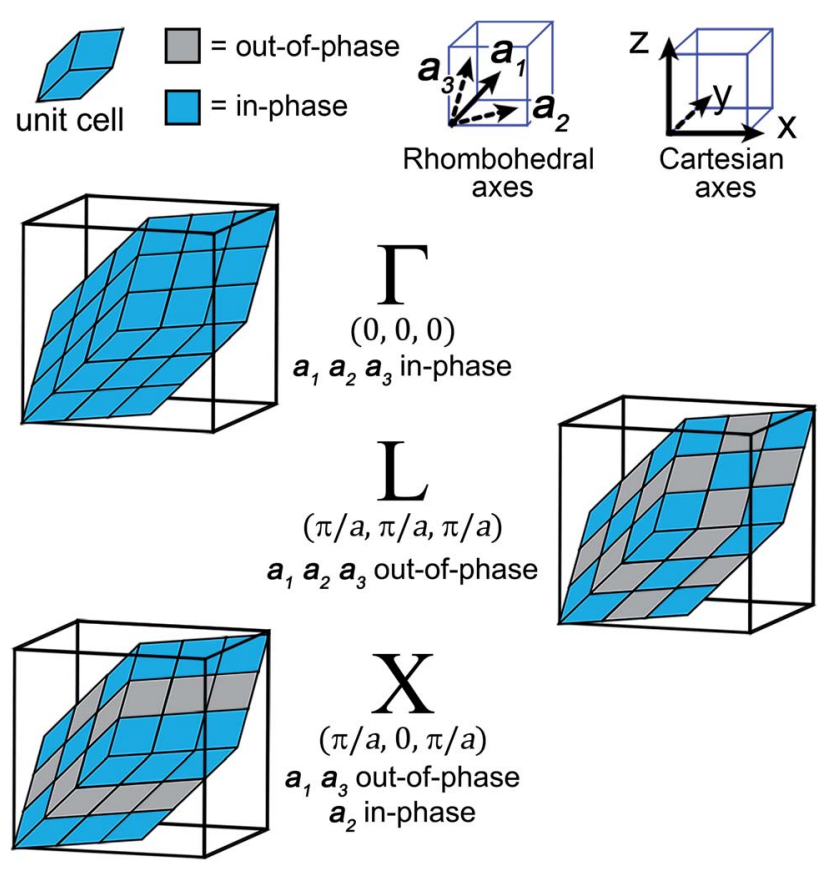

Fig. 3 The translational symmetry, in real space, of the high-symmetry $k$ points $(\boldsymbol{\Gamma}, L \& X)$ for the double-perovskite structure.

the rhombohedral vectors allow for propagation of the Bloch wave along the $x y$ (or $y z$ ) plane. Note that the translational symmetry at some $\mathbf{k}$ points results in Bloch waves that lift the degeneracies of formerly degenerate SALCs (the two $E_{g}$ and three $T_{1 u}$ SALCS). In particular, while $\boldsymbol{\Gamma}$ and $\mathbf{L}$ are isotropic $\left(k_{a_{1}}=\right.$ $\left.k_{a_{2}}=k_{a_{3}}\right) X$ is anisotropic $\left(k_{a_{1}}=k_{a_{3}} \neq k_{a_{2}}\right)$, leading to splitting of both the $E_{g}$ and $T_{1 u}$ SALCs at X. Overall, this procedure generates 11 high-symmetry non-degenerate Bloch waves, 3 each at $\boldsymbol{\Gamma}$ and $\mathbf{L}$ and 5 at $\mathbf{X}$ (see Fig. 5).

At high-symmetry $\mathbf{k}$ points, the SALCs around every B site in the lattice will be identical (although the phases may be flipped), and likewise, every $\mathbf{B}^{\prime}$ site will be identical. Note that this is not true for lower-symmetry $\mathbf{k}$ points. Thus, it is sufficient to represent each Bloch wave at high-symmetry $\mathbf{k}$ points by showing the SALCs around just one B and one $\mathrm{B}^{\prime}$ site. Fig. 5 depicts this for all eleven Bloch waves. Complete pictures of the 11 nondegenerate Bloch waves are given in Fig. S5-S17.†

There are several important observations we can make at this point. Inspection of Fig. 5 shows that, although the local symmetry at the B site is set by our initial choice of SALC, the environment around the $\mathrm{B}^{\prime}$ site is generated entirely by the translational symmetry of the double perovskite lattice at the various $\mathbf{k}$ points. Additionally, many of the SALCs that surround the $\mathrm{B}$ and $\mathrm{B}^{\prime}$ sites appear to be a combination of two of the original SALCs $\left(A_{1 g}, T_{1 u}, E_{g}\right)$. Note that, due to the application of translational symmetry, the Bloch waves do not necessarily maintain the degeneracy of their parent SALC (i.e., an $E_{g}$ SALC does not form a doubly degenerate Bloch wave at all $\mathbf{k}$ points). However, to make the connection between the original input SALC and the Bloch wave derived from it explicit, we refer to Bloch waves as "X-derived", where $\mathrm{X}$ is one of the original three 

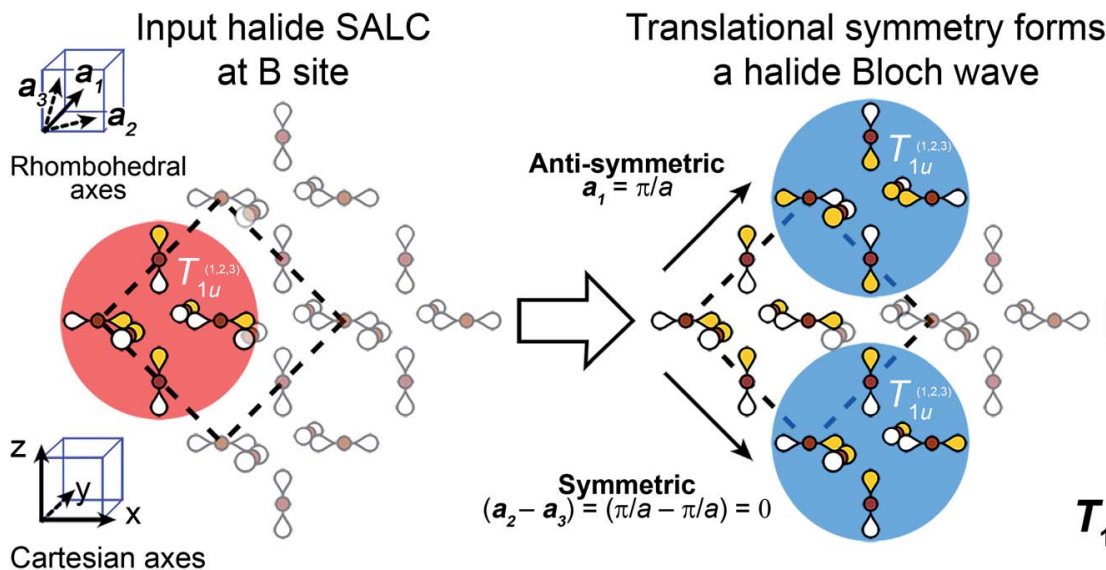
s

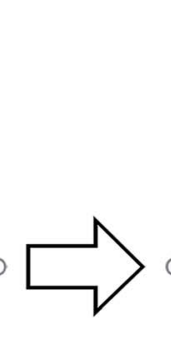
New SALC generated at B' site
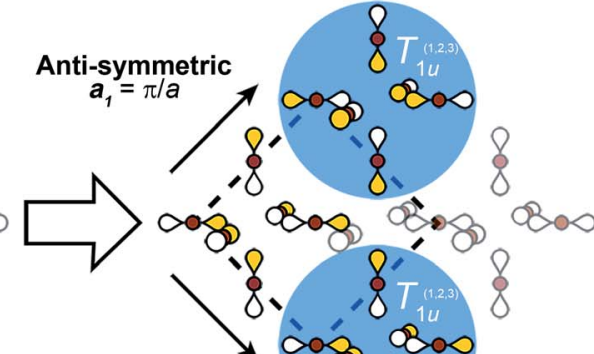

$\left(a_{2}-a_{3}\right)=(\pi / a-\pi / a)=0$

$T_{1 u}: \mathbf{L}\left\{\begin{array}{l}a_{1}=\pi / a \\ a_{2}=\pi / a \\ a_{3}=\pi / a\end{array}\right\}$

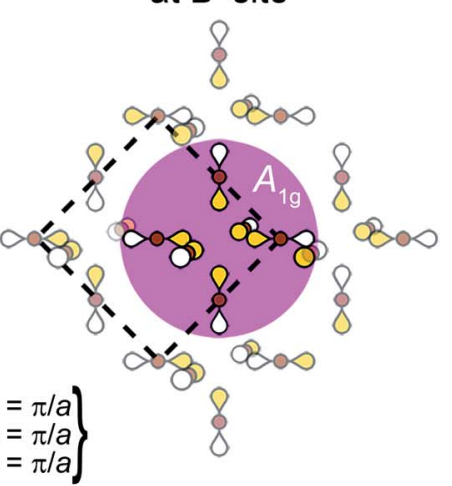

Fig. 4 An example of how to obtain the halide-based Bloch waves at one of the high-symmetry k points of the double-perovskite lattice. The SALC we place at the B site (the "input SALC" highlighted in red, in this example $T_{1 u}$ ) is propagated along the unit-cell axes using the translational symmetry of the $\mathrm{k}$ point (in this example $\mathrm{L}$ ). Here, translational symmetry generates a SALC at the B' site that exhibits $A_{1 g}$ symmetry (the "output SALC", highlighted in pink). Yellow and white lobes represent positive and negative phases, respectively.

SALCs $\left(A_{1 g}, E_{g}, T_{1 u}\right)$. For example, Fig. 4 shows a $T_{1 u}$-derived Bloch wave.

\subsection{Adding the metals and evaluating bonding interactions}

Thus far, we have focused on symmetry and neglected any energetic considerations. Now we consider how the halide-only Bloch waves we have constructed interact with the B-site metal atomic orbitals and use this to rank all the possible Bloch waves on a relative energy scale. Although this approach will work for any set of orbitals, because we are primarily concerned with the valence band maximum (VBM) and conduction band minimum (CBM), we can limit our discussion to the frontier atomic orbitals of the B and B' elements. This is because, when metal contributions are present, the highest occupied and lowest unoccupied molecular orbitals (HOMO and LUMO, respectively) will give rise to the highest-energy valence and lowestenergy conduction bands, respectively.

Intuitively, the energy of a given Bloch wave is proportional to the sum of all the bonding and anti-bonding interactions it generates throughout the material. The large number of atoms in the unit cell and complex geometry of the structure makes this sum difficult to evaluate fully. Instead, we must decide which interactions have the largest effect on the overall energy and are the most important to consider. Fig. 6 summarizes our key assumptions. The B-X nearest-neighbour interactions ( $\sigma$ bonds) are, by far, the largest contributors to the overall energy. This statement can be justified by observing that $\mathrm{B}-\mathrm{X}$ bonds are the shortest bonds in the perovskite structure and, therefore, large orbital overlap and strong energy-level splitting are expected. The next strongest interactions, although much weaker than $\mathrm{B}-\mathrm{X}$ bonds, are the $90^{\circ} \mathrm{X}-\mathrm{X}$ interactions and the $180^{\circ} \mathrm{X}-\mathrm{X} \sigma$ bonds that occur between the halides surrounding the $\mathrm{B}$ site. Whereas the $90^{\circ} \mathrm{X}-\mathrm{X}$ interaction occurs over a shorter distance, the $180^{\circ} \mathrm{X}-\mathrm{X} \sigma$ bond might be expected to have better overlap due to the head-to-head orientation of the $\mathrm{p}$ orbitals. However, empirically we find that, for chlorides, bromides, and iodides, treating the $90^{\circ} \mathrm{X}-\mathrm{X}$ interaction as the stronger of the two interactions gives the correct results (see Section 3.2). This may differ for fluorides or oxides, which have smaller $\mathrm{p}$ orbitals and lattice spacings. Evaluating the energy of each Bloch wave considering just these $\mathrm{B}-\mathrm{X}$ and $90^{\circ} \mathrm{X}-\mathrm{X}$ interactions leads to correct predictions of the CBM and VBM of the vast majority of double perovskite band structures. This approach is the qualitative equivalent of a tight-binding model, which includes both nearest-neighbour and second-nearest-neighbour interactions.

\section{Results}

To demonstrate our methodology, we now present two case studies of known perovskites. These studies represent cases where the material's band extrema are determined by $\mathrm{B}-\mathrm{X}$ interactions only, $\mathrm{X}-\mathrm{X}$ interactions only, or a combination of $\mathrm{B}-\mathrm{X}$ and $\mathrm{X}-\mathrm{X}$ interactions. These three situations cover all possibilities for cubic double perovskites and the rules we develop for them can be generalized to predict the extrema of double perovskites with any combination of B-site cations. We then evaluate the success of our LCAO theory against a large number of known perovskites.

\subsection{Case study 1: perovskites where both B-site frontier orbitals participate in the band}

Summary: in perovskites where both the B- and B'-site metal orbitals contribute to a band, the conduction band minimum or valence band maximum occurs at the $\mathbf{k}$ point where the symmetry of the halide SALCs around the B and B' sites match the symmetry of the metal frontier orbitals at both sites.

When both the B and $\mathrm{B}^{\prime}$ elements have orbitals that participate in a band, our assumption that B-X interactions largely determine the relative energies of the Bloch waves at the highsymmetry $\mathbf{k}$ points is valid. It is instructive to use $\mathrm{Cs}_{2} \mathrm{AgBiBr}_{6}$ as an example of this case. The indirect bandgap of this 


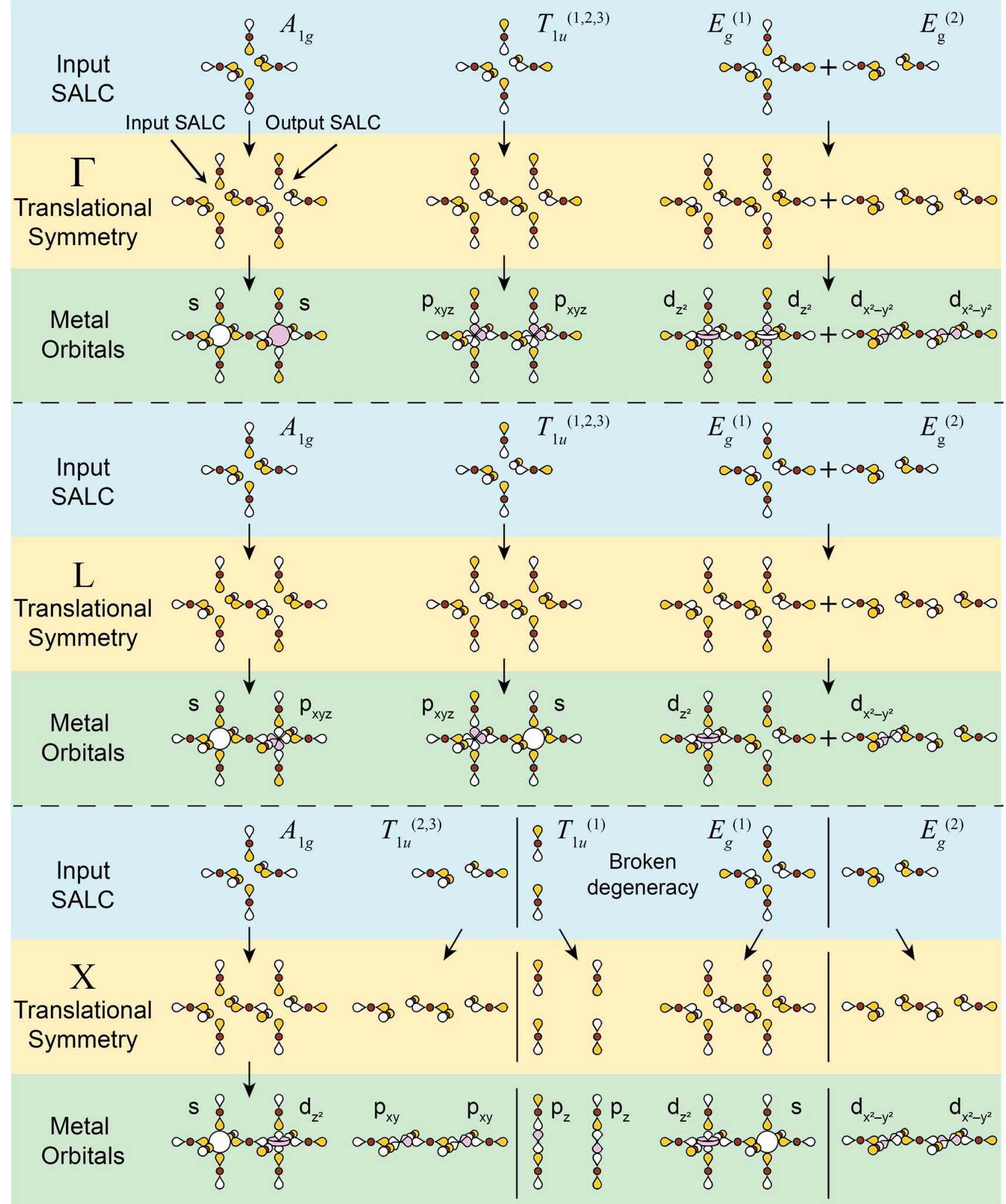

Fig. 5 Summary of all possible Bloch waves for the $\sigma$-bonding halide SALCs at the high-symmetry k points $\Gamma$, L, and $\mathrm{X}$ and their interactions with metal-centered orbitals. The "input SALC" is shown on the left side and the "output SALC", which is generated by translational symmetry (see Fig. 4 for further explanation), is shown on the right side. Here, only one unit cell is shown, but all other unit cells will have the same symmetry. Colored and empty lobes represent positive and negative phases respectively. For ease of visualization, the three degenerate $T_{1 u}$ SALCs $\left(T_{1 u}^{(1)}, T_{1 u}^{(2)}, T_{1 u}^{(3)}\right)$ and their associated Bloch waves are shown together whereas the two degenerate $E_{g} \operatorname{SALCs}\left(E_{g}^{(1)}, E_{g}^{(2)}\right)$ and their associated Bloch waves are shown separately. Both degenerate sets $\left(E_{g}\right.$ and $\left.T_{1 u}\right)$ split at $\mathrm{X}$ with the new non-degenerate SALCs and associated Bloch waves separated by solid black lines. Metal orbitals are assigned by inspecting the symmetry around the $B$ and $B^{\prime}$ sites. 


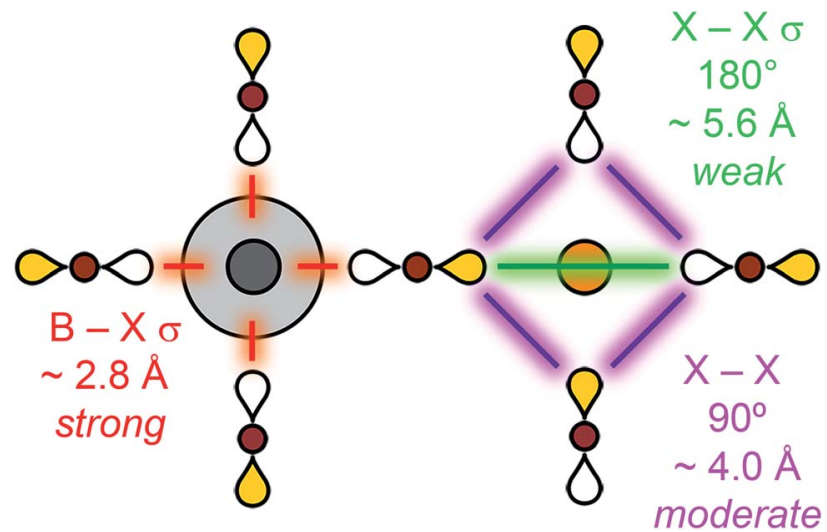

Fig. 6 Hierarchy of bonding interactions in a halide perovskite lattice. Distances are given for $\mathrm{Cs}_{2} \mathrm{AgBiBr}_{6}{ }^{10} \mathrm{~B}=$ metal, $\mathrm{X}=\mathrm{Cl}, \mathrm{Br}$ or I.

perovskite has been experimentally and theoretically verified by several different groups. ${ }^{11-13}$ Using our analysis we can understand the atomistic origins of this indirect transition.

We will begin by examining the valence band. We know that the VBM will occur at one of the high-symmetry $\mathbf{k}$ points, so our task is to determine which of these points has the highest energy. We postulate that the frontier orbitals of both metals $\left(\mathrm{Ag}^{+} 4 \mathrm{~d}_{z^{2}} / 4 \mathrm{~d}_{x^{2}-y^{2}}\right.$ and $\left.\mathrm{Bi}^{3+} 6 \mathrm{~s}\right)$ have sufficiently similar energies to contribute to the valence band (Section 3.2 will address perovskites where this is not the case). For cations with the valence electron configuration of $4 \mathrm{~d}^{10} 5 \mathrm{~s}^{0}\left(\mathrm{Ag}^{+}\right)$or $6 \mathrm{~s}^{2} 6 \mathrm{p}^{0}\left(\mathrm{Bi}^{3+}\right)$, a simple octahedral MO diagram (Fig. $\mathrm{S} 1 \dagger$ ) indicates that the HOMO (and therefore the VBM) will have antibonding character. Therefore, to find the highest-energy $\mathbf{k}$ point of the valence band, we simply need to find which of the Bloch waves in Fig. 5 maximizes the number of antibonding interactions between the halide SALCs and the metal orbitals. A metal orbital will form bonding or antibonding interactions with a halide SALC that matches its symmetry and a nonbonding interaction with a SALC that does not. Since both filled $\mathrm{Bi}^{3+} 6 \mathrm{~s}$ and $\mathrm{Ag}^{+} 4 \mathrm{~d}$ orbitals participate in the valence band, the Bloch wave with maximal antibonding interactions should involve SALCs that match the symmetry of the $\mathrm{s}$ and $\mathrm{d}_{z^{2}} / \mathrm{d}_{x^{2}-y^{2}}$ orbitals. Inspection of Fig. 5 shows that two Bloch waves, both at $\mathbf{X}$, simultaneously match the symmetry of the $\mathrm{Bi}^{3+} 6 \mathrm{~s}$ and the $\mathrm{Ag}^{+} 4 \mathrm{~d}_{z^{2}}$ orbitals implying that the VBM will be at $\mathbf{X}$ (Fig. 7B). Due to the splitting of the $E_{g^{-}}$-derived Bloch waves at $\mathbf{X}$, the $\mathrm{Ag}^{+} 4 \mathrm{~d}_{x^{2}-y^{2}}$ orbital forms a separate band that has fewer overall antibonding interactions and is, therefore, not the VBM.

A similar analysis is possible for the CBM. Again, we assume that the $\mathrm{B}$ and $\mathrm{B}^{\prime}$ frontier orbitals (empty $\mathrm{Bi}^{3+} 6 \mathrm{p}$ and $\mathrm{Ag}^{+} 5 \mathrm{~s}$ orbitals) both participate in the band. We know from the simple octahedral MO diagram (Fig. S1 $\dagger$ ) that the LUMO is antibonding, meaning that the conduction band will be antibonding overall. We are seeking the lowest-energy $\mathbf{k}$ point for the CBM; therefore, we should maximize bonding interactions while maintaining an overall antibonding character to the band. Because both metals contribute to the CBM, the lowest-energy net-antibonding state is one where one metal is bonding and the other is anti-bonding with the halides. As long as the antibonding metal contributes more to the electronic state, the band will remain net antibonding. Importantly, we do not need to know which metal is antibonding and which is bonding with the halides in order to correctly predict the CBM since both bonding and antibonding interactions require a symmetry match between the metal orbital and SALC. As for the VBM, we simply need to determine which Bloch wave has SALCs that match the orbital symmetry of both metals. In this case, the symmetry of the $\mathrm{Bi}^{3+} 6 \mathrm{p}$ and $\mathrm{Ag}^{+} 5$ s orbitals are matched by two Bloch waves, which both occur at $\mathbf{L}$, indicating that the CBM is at $\mathbf{L}$ (Fig. 7A).

We can check our results against the band structure of $\mathrm{Cs}_{2}-$ $\mathrm{AgBiBr}_{6}$ computed through DFT (Fig. 7C). Consistent with our model's predictions, the VBM lies at $\mathbf{X}$ and the CBM at $\mathbf{L}$, yielding the experimentally observed indirect gap. The band structure can be deconstructed with atomic detail by projecting the electronic states from the DFT calculation onto spherical harmonics centered at each metal ion (plotted in color in Fig. 7C). We can view each individual band as arising from a root metal-hexahalide $\left(\mathrm{BX}_{6}{ }^{n-}\right)$ molecular orbital at the $\mathrm{B}$ site, whose energy is perturbed by the additional metal-halide interactions around the $\mathrm{B}^{\prime}$ site (or vice versa). For the two highest-energy valence bands, Fig. 7C shows $\mathrm{Ag} d$ character present at all values of $\mathbf{k}$. This means that the MOs formed from an antibonding combination of $\mathrm{Ag} 4 \mathrm{~d}_{z^{2}}$ or $4 \mathrm{~d}_{x^{2}-y^{2}}$ atomic orbitals with the $E_{g}$ SALCs can be considered as the root MOs that form these bands. One of these MOs will be a component of every electronic state in the upper two valence bands. Reading down the $E_{g}$ column in Fig. 5 gives the Bloch waves for these bands at $\boldsymbol{\Gamma}, \mathbf{L}$, and $\mathbf{X}$ (Fig. 7B; $\mathbf{L}$ not shown). From Fig. 5 , the $\mathrm{d}_{z^{2}}$ and $\mathrm{d}_{x^{2}-y^{2}}$ bands are degenerate at $\boldsymbol{\Gamma}$ and split at $\mathbf{X}$, which is reproduced in the band structure. In both bands, the SALC around the Ag site remains constant, while the SALC around the Bi site changes with $\mathbf{k}$. For example, in the $\mathrm{Ag}_{z^{2}}$ band along the $\boldsymbol{\Gamma}$ to $\mathbf{X}$ direction, the Bi-centered SALC gradually transitions from $E_{g}$ symmetry to $A_{1 g}$ symmetry and its interaction with the Bi 6 s orbital changes from non-bonding at $\Gamma$ to antibonding at $\mathbf{X}$ (Fig. 5). This increased antibonding character pushes the energy at $\mathbf{X}$ above that at $\boldsymbol{\Gamma}$ to form the VBM at $\mathbf{X}$. Consistent with this prediction, Fig. 7C shows Bi s character only near $\mathbf{X}$, coincident with an upturn in the $\mathrm{Ag} \mathrm{d}_{z^{2}}$ band's energy. In contrast, the $\mathrm{Ag}$ $\mathrm{d}_{x^{2}-y^{2}}$ band remains nonbonding with the Bi $6 \mathrm{~s}$ orbital from $\Gamma$ to $\mathbf{X}$ and is energetically flat. According to Fig. 5, the two bands should be degenerate at $\mathbf{L}$, but they are split in the DFT band structure due to spin-orbit coupling (see Fig. S3 and discussion in the ESI $\dagger$ ).

We can perform a similar analysis for the conduction bands. Here the presence of Bi $\mathrm{p}$ character at all $\mathbf{k}$ points in Fig. 7A indicates that these bands' root MOs are the antibonding combinations of the three Bi $6 \mathrm{p}$ orbitals with the $T_{1 u}$ symmetry halide SALCs, leading to the observation of Bi p character at all k points in Fig. 7C. From the $T_{1 u}$ column of Fig. 5, we find that, moving from $\boldsymbol{\Gamma}$ to $\mathbf{L}$, the symmetry of the SALC at the Ag site gradually changes from $T_{1 u}$ symmetry to $A_{1 g}$ symmetry (Fig. 7A). As the symmetry of the SALC approaches $A_{1 g}$, the bonding interaction between the SALC and the Ag $5 \mathrm{~s}$ orbital becomes 


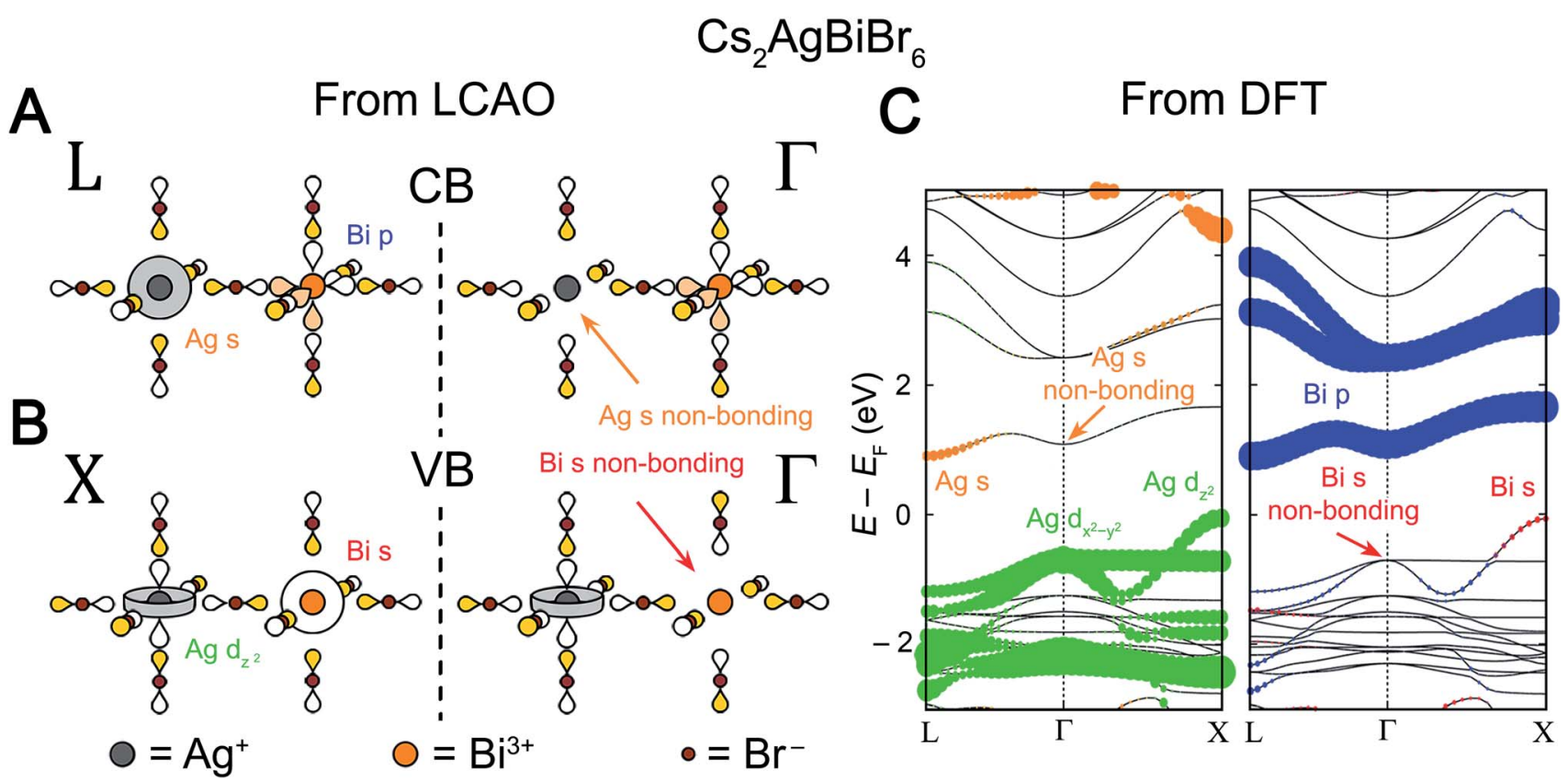

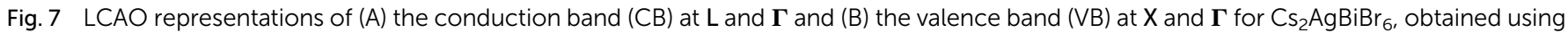
the LCAO theory illustrated in Fig. 5. Net nonbonding interactions between the halide SALCs and the metal s orbitals explain the missing Ag $5 s^{\circ}$ (orange arrow) and $\mathrm{Bi} 6 \mathrm{~s}^{2}$ (red arrow) orbital contributions at $\boldsymbol{\Gamma}$ at the $\mathrm{CB}$ and $\mathrm{VB}$, respectively. Colored and empty lobes represent positive and negative phases, respectively. (C) Band structure of the double perovskite $\mathrm{Cs}_{2} \mathrm{AgBiBr}_{6}$ computed using DFT, showing an indirect gap from $\mathrm{X}$ to $\mathrm{L}$. The band structure is shown in duplicate and the orbital contributions of the B-site atoms ( $\mathrm{Ag}$ and Bi) are given in color with the dot size proportional to the size of the orbital contribution. Bromide contributions are present but not shown. Note that Ag $5 \mathrm{~s} c h a r a c t e r$ is absent at the $\boldsymbol{\Gamma}$ point in the CB and that Bi 6 s character is absent at the $\boldsymbol{\Gamma}$ point in the VB (denoted by orange and red arrows, respectively), in agreement with the LCAO analysis.

more significant, lowering the energy of the band at $\mathbf{L}$ relative to that at $\boldsymbol{\Gamma}$ and forming the CBM at $\mathbf{L}$. Notice again that while the band is derived from a Bi-centered MO, the dispersion (the variation of a band's energy with $\mathbf{k}$ ) is determined by changes in the bonding around the $\mathrm{Ag}$ site. The three conduction bands in Fig. 7C (derived from mixtures of $\mathrm{p}_{x}, \mathrm{p}_{y}$, and $\mathrm{p}_{z}$ orbitals) are not degenerate at $\boldsymbol{\Gamma}$ and $\mathbf{L}$ although they are expected to be from Fig. 5. As in the valence band, this is due to spin-orbit coupling (see Fig. S3 and discussion in the ESI†).

DFT provided information about which orbital was present at all $\mathbf{k}$ points within a given band (and therefore was the root $\mathrm{MO}$ ) and which was present at only one, enabling us to develop a detailed and intuitive description of band dispersion in $\mathrm{Cs}_{2}-$ $\mathrm{AgBiBr}_{6}$. However, we were able to correctly predict the VBM/ CBM without utilizing DFT, relying on qualitative arguments alone. In general, if both orbitals participate in the band edges, determining the VBM/CBM only requires knowledge of the frontier orbitals. The results, for every possible combination of $\mathrm{B}$ and $\mathrm{B}^{\prime}$ orbitals, are summarized in Table 1 . Here, the conduction band and the valence band should be evaluated independently. For example, using Table 1 for $\mathrm{Cs}_{2} \mathrm{AgBiBr}_{6}$, the filled $\mathrm{Ag}$ and $\mathrm{Bi}$ frontier orbitals that form the valence band are $\mathrm{d}$ and s orbitals, respectively, affording a maximum at $\mathbf{X}$. Similarly, empty $\mathrm{Ag} \mathrm{s}$ and $\mathrm{Bi} \mathrm{p}$ orbitals compose the conduction band, generating a minimum at $\mathbf{L}$.

\subsection{Case study 2: perovskites where at least one B-site frontier orbital does not participate in the band}

Summary: in perovskites where only one metal contributes to the band, the conduction band minimum or valence band maximum is set by the $90^{\circ}$ interactions of the halides surrounding the other (nonparticipating) metal site.

The analysis in Section 3.1 requires both B-site atoms to have orbitals that participate in the bands near the bandgap. However, there are several examples of double perovskites where one of the $\mathrm{B}$ sites is empty, as in $\mathrm{A}_{2} \mathrm{~B}^{\mathrm{IV}} \square \mathrm{X}_{6}(\square=$ vacancy), ${ }^{38-42}$ or where the orbitals of one or both of the B-site HOMOs/LUMOs are of incorrect energy to be near the bandgap (for example the valence band of $\mathrm{Cs}_{2} \mathrm{AgTlX}_{6}$ ). ${ }^{17}$ We use the example of $\mathrm{Cs}_{2} \mathrm{Sn}^{\mathrm{IV}} \square \mathrm{Br}_{6}$ to illustrate how to use weaker $\mathrm{X}-\mathrm{X}$ interactions to obtain the correct band structure in these cases.

The $\mathrm{Sn}^{4+}$ LUMO is the $5 \mathrm{~s}$ orbital and, from Fig. S1, $\dagger$ we expect it to be a major component of the lowest-energy $\mathrm{Cs}_{2} \mathrm{Sn}^{\mathrm{IV}} \square \mathrm{Br}_{6}$ conduction band. To match the Sn 5s orbital symmetry, the Bloch waves of this conduction band must be derived from the $A_{1 g}$ SALC. Reading down the $A_{1 g}$ column in Fig. 5 gives the Bloch waves for the $\mathrm{Cs}_{2} \mathrm{Sn}^{\mathrm{IV}} \square \mathrm{Br}_{6}$ conduction band at $\boldsymbol{\Gamma}$, $\mathbf{L}$, and $\mathbf{X}$ (Fig. 8A). Because the SALC around the Sn site is the same in all three of these Bloch waves, each has the same number of $\mathrm{B}-\mathrm{X}$ antibonding interactions. In fact, the three Bloch waves only differ in what SALC is generated around the vacant $\mathrm{B}^{\prime}$ site where 
B-X interactions are not possible. Therefore, to understand the dispersion of this band and energetically distinguish the three $\mathbf{k}$ points, we must consider how the weaker $\mathrm{X}-\mathrm{X}$ interactions affect the energy of each Bloch wave.

There are two possible $\mathrm{X}-\mathrm{X}$ interactions that may be significant: the $180^{\circ} \mathrm{X}-\mathrm{X} \sigma$ interaction and the $90^{\circ} \mathrm{X}-\mathrm{X}$ interaction (Fig. 6). We will consider three limiting scenarios. First, that only the $180^{\circ}$ interactions are important, second that only the $90^{\circ} \mathrm{X}-\mathrm{X}$ interactions are important, and third that both the $180^{\circ}$ and $90^{\circ}$ interactions are equally important. The relative energies of the three SALCs surrounding the vacant site in Fig. 8A can be evaluated based on the number of bonding and antibonding interactions they produce under each of these assumptions (Fig. S18 $\dagger$ ). For a stronger $180^{\circ} \mathrm{X}-\mathrm{X} \sigma$ interaction, the conduction band Bloch waves at the high-symmetry $\mathbf{k}$ points are ranked from lowest to highest energy as follows: $\boldsymbol{\Gamma}=\mathbf{X}<\mathbf{L}$. Assuming a stronger $90^{\circ} \mathrm{X}-\mathrm{X}$ interaction gives: $\boldsymbol{\Gamma}<\mathbf{L}<\mathbf{X}$. If both interactions are equally significant, the ordering becomes $\mathbf{\Gamma}<\mathbf{X}$ $<\mathbf{L}$. To distinguish between these scenarios, we use information from DFT.

The DFT band structure of $\mathrm{Cs}_{2} \mathrm{Sn}^{\mathrm{IV}} \square \mathrm{Br}_{6}$ is shown in Fig. 8C with the Sn contributions shown in color. As expected, the Sn $5 \mathrm{~s}$ orbital is present at all $\mathbf{k}$ points in the conduction band. The relative energies of the conduction band Bloch waves in Fig. 8C $(\boldsymbol{\Gamma}<\mathbf{L}<\mathbf{X})$ is consistent with the $90^{\circ} \mathrm{X}-\mathrm{X}$ interaction being stronger than the $180^{\circ} \mathrm{X}-\mathrm{X}$ interaction. In fact, every reported $\mathrm{A}_{2} \mathrm{~B}^{\mathrm{IV}} \square \mathrm{X}_{6}$ perovskite electronic structure $\left(\mathrm{B}^{\mathrm{IV}}=\mathrm{Sn}^{4+}, \mathrm{Pd}^{4+}, \mathrm{Hf}^{4+}\right.$,
$\left.\mathrm{Te}^{4+}, \mathrm{Ti}^{4+}\right)^{38-42}$ supports the conclusion that the $90^{\circ} \mathrm{X}-\mathrm{X}$ interaction is, after $\mathrm{B}-\mathrm{X}$ interactions, the most important.

In some double perovskites, no metal orbital has the correct energy to be present near the valence band maximum and the band is composed of pure halide orbitals that are non-bonding with respect to the $\mathrm{B}$-site metals. The valence band of $\mathrm{Cs}_{2}$ $\mathrm{Sn}^{\mathrm{IV}} \square \mathrm{Br}_{6}$ is one such case. The $\mathrm{Sn}^{4+}$ HOMO, a $4 \mathrm{~d}$ orbital, has a binding energy of 23-27 eV below vacuum, as determined by $\mathrm{X}$-ray photoelectron spectroscopy, ${ }^{43}$ too low in energy to participate in bonding. Thus, the bandgap transition is a pure ligandto-metal charge transfer (LMCT). Although there are no $\mathrm{B}-\mathrm{X}$ interactions in the $\mathrm{Cs}_{2} \mathrm{Sn}^{\mathrm{IV}} \square \mathrm{Br}_{6}$ valence band, the $90^{\circ} \mathrm{X}-\mathrm{X}$ interactions are still present, and these alone will determine the VBM. Fig. 8B shows the Bloch waves from Fig. 5 with the most $\mathrm{X}-\mathrm{X}$ antibonding character at $\mathbf{L}, \boldsymbol{\Gamma}$, and $\mathbf{X}$. These Bloch waves correspond to the highest-energy occupied states at each highsymmetry $\mathbf{k}$ point in the valence band. The Bloch wave at $\mathbf{L}$ shows 4 net-antibonding interactions, whereas both $\boldsymbol{\Gamma}$ and $\mathbf{X}$ show 8 net-antibonding interactions. The $E_{g}$-derived Bloch waves at $\boldsymbol{\Gamma}$ and $\mathbf{X}$, thus produce the VBM. While the valence band Bloch waves at $\boldsymbol{\Gamma}$ and $\mathbf{X}$ have the same number of net antibonding interactions, in the DFT band structure of $\mathrm{Cs}_{2}$ $\mathrm{Sn}^{\mathrm{IV}} \square \mathrm{I}_{6}, \mathbf{X}$ lies slightly below $\boldsymbol{\Gamma}$. This can be justified by noting that antibonding interactions are slightly more destabilizing than their corresponding bonding interactions are stabilizing. This should push $\boldsymbol{\Gamma}$ slightly higher than $\mathbf{X}$ as it has more antibonding interactions overall. However, this phenomenon does not appear to be general; other compounds with halide-only

\section{$\mathrm{Cs}_{2} \mathrm{Sn}^{\mathrm{IV}} \mathrm{Br}_{6}$}
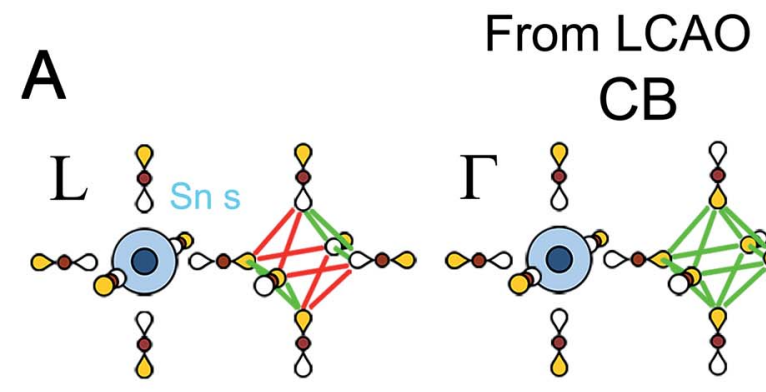

B
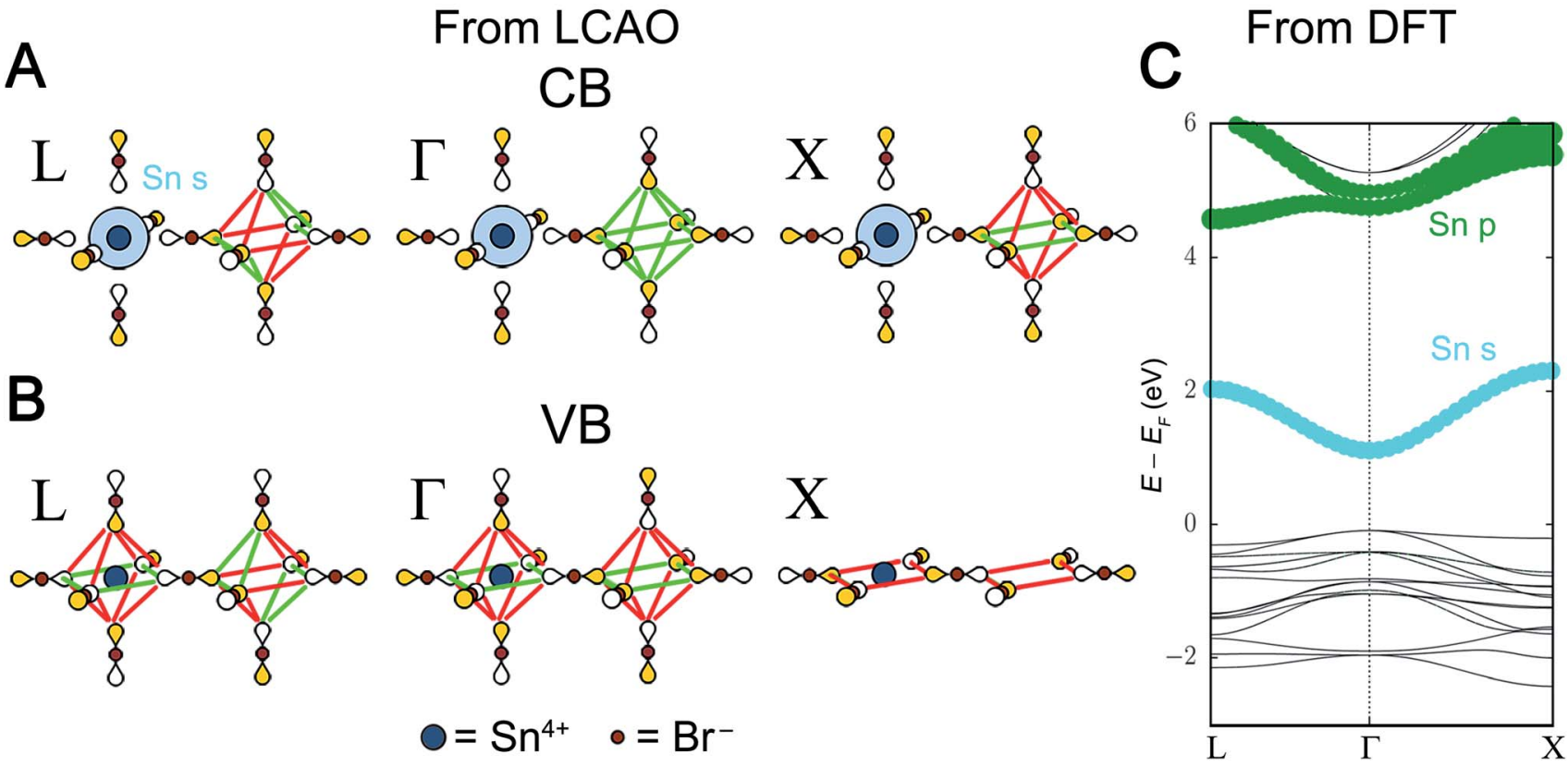

Fig. 8 LCAO representations of (A) the conduction band (CB) and (B) valence band (VB) of $\mathrm{Cs}_{2} \mathrm{Sn}^{\mathrm{IV}} \square \mathrm{Br}_{6}$ obtained from Fig. 5. Colored lobes represent positive phases and empty lobes represent negative phases. Green and red lines show the $90^{\circ} \mathrm{X}-\mathrm{X}$ bonding and antibonding interactions, respectively, between the bromide p orbitals. (C) DFT band structure of the double perovskite $\mathrm{Cs}_{2} \mathrm{Sn}^{\mathrm{IV}} \square \mathrm{Br}_{6}$. The orbital contributions of the $\mathrm{Sn}$ atom are given in color with dot size proportional to the size of orbital contribution. Bromide contributions are present but not shown. 
valence bands ( $\mathrm{Cs}_{2} \mathrm{Hf}^{\mathrm{IV}} \square \mathrm{I}_{6}, \mathrm{Cs}_{2} \mathrm{Pd}^{\mathrm{IV}} \square \mathrm{Br}_{6}, \mathrm{Cs}_{2} \mathrm{Ti}^{\mathrm{IV}} \square \mathrm{I}_{6}$ ) show a much closer match between $\boldsymbol{\Gamma}$ and $\mathbf{X}^{\mathbf{4 0 - 4 2}}$ We collect predictions for all possible perovskite compositions where only one Bsite orbital or no B-site orbitals participate in the band edges in Table 1.

Finally, we note that the diagrams we construct in Fig. 8A and $\mathrm{B}$ contain additional information beyond the relative energies of the high-symmetry $\mathbf{k}$ points. For example, $\mathbf{C s}_{2^{-}}$ $\mathrm{Sn}^{\mathrm{IV}} \square \mathrm{Br}_{6}$ is isostructural and isoelectronic with $\mathrm{Cs}_{2} \mathrm{Sn}^{\mathrm{IV}} \square \mathrm{I}_{6}$, which is known to possess a parity-forbidden bandgap. ${ }^{39}$ Parity-forbidden bandgaps have been identified in several other double perovskites, ${ }^{17,44}$ and are an emerging topic of interest. While treating this problem exhaustively is beyond the scope of our current work, we can see from Fig. 8 how the parity-forbidden bandgap arises in $\mathrm{Cs}_{2} \mathrm{Sn}^{\mathrm{IV}} \square \mathrm{I}_{6}$. Inspection of Fig. $8 \mathrm{~A}$ and $\mathrm{B}$ at $\Gamma$ show that, around both the B and $\mathrm{B}^{\prime}$ site, the CBM consists of $A_{1 g}$ symmetry SALCs and the VBM consists of $E_{g}$ symmetry SALCs. The gerade symmetry of both the VBM and CBM states means that an optical transition between them is parity (or Laporte) forbidden.

\section{Discussion}

\subsection{Rules for determining band extrema}

We have now treated all possible scenarios involving perovskites with $\sigma$-bonding orbitals at their band edges (main group and $\mathrm{d}^{10}$ cations). A similar analysis for perovskites with $\pi$ bonding orbitals at their band edges $\left(\mathrm{d}^{0}-\mathrm{d}^{9}\right.$ cations $)$ is given in the ESI. $\dagger$ It is worth pausing at this point to summarize our results and benchmark them to literature DFT reports of double

Table 1 Expected $\mathrm{k}$ points of the conduction band minimum (CBM) and valence band maximum (VBM) for all possible combinations of $B$ and $\mathrm{B}^{\prime}$ orbitals. Note that the VBM and CBM must be evaluated separately for a given perovskite

\begin{tabular}{|c|c|c|c|}
\hline \multicolumn{2}{|l|}{ Orbitals } & \multicolumn{2}{|c|}{ Prediction } \\
\hline B & $\mathrm{B}^{\prime}$ & VBM & CBM \\
\hline \multicolumn{4}{|c|}{$\sigma$-Bonding states ${ }^{a}$} \\
\hline $\mathrm{s}$ & $\mathrm{s}$ & $\Gamma$ & $\Gamma$ \\
\hline $\mathrm{p}$ & $\mathrm{p}$ & $\Gamma$ & $\Gamma$ \\
\hline $\mathrm{d}_{x^{2}-y^{2}} / \mathrm{d}_{z^{2}}$ & $\mathrm{~d}_{x^{2}-y^{2}} / \mathrm{d}_{z^{2}}$ & $\Gamma \& \mathbf{X}$ & $\Gamma \& \mathrm{X}$ \\
\hline $\mathrm{s}$ & $\mathrm{p}$ & $\mathbf{L}$ & $\mathbf{L}$ \\
\hline s & $\mathrm{d}_{x^{2}-y^{2}} / \mathrm{d}_{z^{2}}$ & $\mathbf{X}$ & $\mathbf{X}$ \\
\hline $\mathrm{p}$ & $\mathrm{d}_{x^{2}-y^{2}} / \mathrm{d}_{z^{2}}$ & - & - \\
\hline $\mathrm{s}$ & Null & $\mathbf{X}$ & $\Gamma$ \\
\hline $\mathrm{p}$ & Null & $\mathbf{L}$ & $\mathbf{L}$ \\
\hline $\mathrm{d}_{x^{2}-y^{2}} / \mathrm{d}_{z^{2}}$ & Null & $\Gamma \& \mathbf{X}$ & $\mathbf{x}$ \\
\hline Null & Null & $\Gamma \& \mathbf{X}$ & - \\
\hline \multicolumn{4}{|c|}{$\pi$-Bonding states ${ }^{a}$} \\
\hline $\mathrm{d}_{x y / y z / x z}$ & $\mathrm{~d}_{x y / y z / x z}$ & $\Gamma \& \mathbf{X}$ & $\Gamma \& X$ \\
\hline $\mathrm{d}_{x y / y z / x z}$ & Null & $\mathbf{X}$ & $\Gamma \& X$ \\
\hline Null & Null & $\boldsymbol{\Gamma}^{b}$ & - \\
\hline
\end{tabular}

${ }^{a} \pi$ - and $\sigma$-bonding states involve orthogonal orbitals and cannot interact (see ESI). ${ }^{b}$ The $\sigma$-bonding halide-only states have more $90^{\circ}$ $\mathrm{X}-\mathrm{X}$ interactions than the $\pi$-bonding ones and will often form the band extrema (see ESI). perovskites (Section 4.2). From the case studies, we have developed three rules for determining band extrema:

1. If both $\mathrm{B}$ and $\mathrm{B}^{\prime}$ metal orbitals contribute substantially to the band, the band extremum will be at the $\mathbf{k}$ point where the halide SALCs around each site match the symmetry of the B and $\mathrm{B}^{\prime}$ HOMOs (for the VBM) or LUMOs (for the CBM).

2. If only one B-site metal orbital is significantly involved in a band, the VBM will be at the $\mathbf{k}$ point with SALCs that match the symmetry of the HOMO around the participating metal and maximize the $90^{\circ} \mathrm{X}-\mathrm{X}$ antibonding interactions around the non-participating metal site. Similarly, the CBM will be at the $\mathbf{k}$ point with SALCs that match the symmetry of the LUMO around the participating metal and maximize the $90^{\circ} \mathrm{X}-\mathrm{X}$ bonding interactions around the non-participating metal site.

3. If no B-site metal orbitals participate in the valence band, the VBM will occur at the $\mathbf{k}$ point that maximizes the number of $90^{\circ} \mathrm{X}-\mathrm{X}$ antibonding interactions around both sites. This will always be at the $\boldsymbol{\Gamma}$ and $\mathbf{X}$ points.

Following these rules allows for predictions of band extrema for all possible combinations of orbitals. These predictions are summarized in Table 1.

\subsection{Comparison of the theory to experiment and calculations}

For the LCAO theory to be successful, it must accurately predict the $\mathbf{k}$ point of both the VBM and CBM for the full range of double perovskites. In Table 2, we compare the predictions contained in Table 1 to predictions determined by DFT-based methods for a wide range of known cubic double perovskites. To ensure consistency in making this comparison, we use the computed density of states to determine the orbital composition of the valence and conduction bands of each compound. In many cases, this additional information is unnecessary as determining the orbital composition of these bands from chemical reasoning or experimental measurement of orbital energies results in the correct answer. The LCAO theory is remarkably successful, showing full agreement with DFT results for all synthesized double perovskite compositions of which we are aware, except for one. The exception is the valence band of $\mathrm{Cs}_{2} \mathrm{AgYCl}_{6}$. Here, the reported density of states shows predominantly $\mathrm{Ag}$ orbital character in the VBM and a band maximum at $\mathbf{L}$, instead of a maximum at $\boldsymbol{\Gamma}$ or $\mathbf{X}$ as our model predicts for a pure $\mathrm{Ag} \mathrm{d}$ band. Nevertheless, the overwhelming agreement between our predictions and computational methods instils confidence in the validity of our approach.

\subsection{Limitations of the theory}

The major limitation of the LCAO approach is that prior knowledge of the orbital compositions of the bands is necessary to obtain accurate results. In our construction of Table 2, we used the calculated wavefunction projections to determine the orbital composition of the bands, but ideally, the theory would require no computational inputs. In fact, for many double perovskites, an experienced chemist can accurately guess the orbital compositions of the band, and therefore the band extrema using the three rules presented here, using chemical 
Table 2 Comparison of the band extrema predicted in Table 1 to those predicted by DFT calculations for a wide range of cubic double perovskites. The B and $\mathrm{B}^{\prime}$ orbitals that contribute to a band were determined from DFT density-of-states calculations but, in principle, could come from chemical reasoning or experimental information. For a given perovskite, the $\mathrm{k}$ points corresponding to band extrema that are calculated to be within $0.1 \mathrm{eV}$ of each other are listed together

\begin{tabular}{|c|c|c|c|c|c|c|c|c|c|c|}
\hline \multirow[b]{2}{*}{ Compound } & \multicolumn{4}{|c|}{ Valence band maximum } & \multicolumn{4}{|c|}{ Conduction band minimum } & \multirow[b]{2}{*}{ Bandgap } & \multirow[b]{2}{*}{ Reference } \\
\hline & $\mathrm{B}$ & $\mathrm{B}^{\prime}$ & Pred. & Calc. & $\mathrm{B}$ & $\mathrm{B}^{\prime}$ & Pred. & Calc. & & \\
\hline $\mathrm{Cs}_{2} \mathrm{AgBiBr}_{6}{ }^{a}$ & $\operatorname{Ag} 4 \mathrm{~d}_{\mathrm{z}^{2}}$ & Bi $6 s$ & $\mathbf{X}$ & $\mathbf{X}$ & $\mathrm{Ag} 5 \mathrm{~s}$ & Bi 6p & $\mathbf{L}$ & $\mathbf{L}$ & Indirect & 12 \\
\hline $\mathrm{Cs}_{2} \mathrm{AgInCl}_{6}$ & $\mathrm{Ag} 4 \mathrm{~d}_{\mathrm{z}^{2}}^{b}$ & Null & $\Gamma$ & $\Gamma$ & $\mathrm{Ag} 5 \mathrm{~s}$ & In $5 \mathrm{~s}$ & $\Gamma$ & $\Gamma$ & Direct & 18 \\
\hline $\mathrm{Cs}_{2} \mathrm{AgSbBr}_{6}{ }^{a}$ & $\mathrm{Ag} 4 \mathrm{~d}_{\mathrm{z}^{2}}$ & $\mathrm{Sb} 5 \mathrm{~s}$ & $\mathbf{X}$ & $\mathbf{X}$ & $\operatorname{Ag} 5 \mathrm{~s}$ & $\mathrm{Sb} 5 \mathrm{p}$ & $\mathbf{L}$ & $\mathbf{L}$ & Indirect & 46 \\
\hline $\mathrm{Cs}_{2} \mathrm{AgTlBr}_{6}{ }^{a}$ & $\mathrm{Ag} 4 \mathrm{~d}_{\mathrm{z}^{2}}{ }^{b}$ & Null & $\Gamma$ & $\Gamma$ & $\operatorname{Ag} 5 \mathrm{~s}$ & $\operatorname{Tl} 6 \mathrm{~s}$ & $\Gamma$ & $\Gamma$ & Direct & 17 \\
\hline $\mathrm{Cs}_{2} \mathrm{AgYCl}_{6}$ & Ag $4 \mathrm{~d}_{\mathbf{z}^{2}}{ }^{b}$ & Null & $\Gamma \& \mathbf{X}$ & $\mathbf{L}$ & $\mathrm{Ag} 5 \mathrm{~s}$ & $\mathrm{Null}^{c}$ & $\Gamma$ & $\Gamma$ & Indirect & 47 \\
\hline $\mathrm{Cs}_{2} \mathrm{KBiCl}_{6}$ & $\mathrm{~K} 3 \mathrm{p}$ & $\mathrm{Bi} 6 \mathrm{~s}$ & $\mathbf{L}$ & $\mathbf{L}$ & $\mathrm{K} 4 \mathrm{p}^{d}$ & Bi $6 p$ & $\Gamma$ & $\Gamma \& L$ & Direct & See ESI \\
\hline $\mathrm{Cs}_{2} \mathrm{KYCl}_{6}$ & Null & Null & $\Gamma \& \mathrm{X}$ & $\Gamma \& X$ & Null & $\mathrm{Y} 4 \mathrm{~d}_{\mathbf{x y}}{ }^{e}$ & $\Gamma$ & $\Gamma$ & Direct & 44 \\
\hline $\mathrm{Cs}_{2} \mathrm{NaBiCl}_{6}$ & Null & $\mathrm{Bi} 6 \mathrm{~s}$ & $\mathbf{X}$ & $\mathrm{X}$ & $\mathrm{Na} 3 \mathrm{p}^{d}$ & Bi $6 \mathrm{p}$ & $\Gamma$ & $\Gamma \& L$ & Indirect & See ESI \\
\hline $\mathrm{Cs}_{2} \mathrm{NaInBr}_{6}{ }^{a, f}$ & Null & Null & $\Gamma \& X$ & $\Gamma \& X$ & Null & In $5 \mathrm{~s}$ & $\Gamma$ & $\Gamma$ & Direct & 48 and 49 \\
\hline$(\mathrm{MA})_{2} \mathrm{TlBiBr}_{6}{ }^{g}$ & $\mathrm{Tl} 6 \mathrm{~s}$ & Bi 6s & $\Gamma$ & $\Gamma$ & $\mathrm{Tl} 6 \mathrm{p}$ & Bi 6p & $\Gamma$ & $\Gamma$ & Direct & 19 \\
\hline $\mathrm{Cs}_{2} \mathrm{TlTlCl}_{6}{ }^{h}$ & $\mathrm{Tl} 6 \mathrm{~s}$ & Null & $\mathbf{X}$ & $\mathrm{X}$ & $\mathrm{Tl} 6 \mathrm{p}$ & $\mathrm{Tl} 6 \mathrm{~s}$ & $\mathbf{L}$ & $\mathbf{L}$ & Indirect & 50 \\
\hline $\mathrm{Cs}_{2} \mathrm{Hf}^{\mathrm{IV}} \square \mathrm{Cl}_{6}{ }^{a}$ & Null & Null & $\Gamma \& X$ & $\Gamma \& X$ & Hf $5 \mathrm{~d}_{\mathbf{x y}}{ }^{e}$ & Null & $\Gamma \& X$ & $\Gamma \& X$ & Indirect & 41 \\
\hline $\mathrm{Cs}_{2} \mathrm{Pd}^{\mathrm{IV}} \square \mathrm{Br}_{6}$ & Null & Null & $\Gamma \& \mathrm{X}$ & $\Gamma \& X$ & $\operatorname{Pd} 4 \mathrm{~d}_{\mathrm{z}^{2}}$ & Null & $\mathbf{X}$ & $\mathbf{X}$ & Direct & 40 \\
\hline $\mathrm{Cs}_{2} \mathrm{Sn}^{\mathrm{IV}} \square \mathrm{I}_{6}{ }^{a}$ & Null & Null & $\Gamma \& \mathrm{X}$ & $\Gamma$ & Sn $5 \mathrm{~s}$ & Null & $\Gamma$ & $\Gamma$ & Direct & 38 \\
\hline $\mathrm{Cs}_{2} \mathrm{Te}^{\mathrm{IV}} \square \mathrm{I}_{6}$ & Te 6s & Null & $\mathbf{X}$ & $\mathbf{X}$ & Te $6 \mathrm{p}$ & Null & $\mathbf{L}$ & $\mathbf{L}$ & Indirect & 39 \\
\hline $\mathrm{Cs}_{2} \mathrm{Ti}^{\mathrm{IV}} \square \mathrm{I}_{6}{ }^{a}$ & Null & Null & $\Gamma \& \mathbf{X}$ & $\Gamma$ & $\operatorname{Ti} 3 \mathrm{~d}_{\mathbf{x y}}{ }^{e}$ & Null & $\Gamma \& \mathbf{X}$ & $\Gamma \& \mathbf{X}$ & Indirect & 42 \\
\hline
\end{tabular}

${ }^{a}$ Isostructural compounds with other halides are known. These compounds are also well-described by the LCAO model. ${ }^{b}$ The $\mathrm{d}_{\mathbf{z}^{2}}$ orbital is degenerate with the $\mathrm{d}_{\mathbf{x}^{2}-\mathbf{y}^{2}}$ orbital. ${ }^{c}$ Bands originating from the $\mathrm{Y}_{4} 4 \mathrm{~d}_{\mathbf{x y}}$ orbitals $\pi$-bonding with the halides are also present near the CBM but are at slightly higher energies and don't interact with the $\sigma$-bonding Ag 5 s states (see ESI). ${ }^{d}$ See ESI for extended discussion of the appearance of alkali $\mathrm{p}$ character in the conduction band of these materials. ${ }^{e}$ The $\mathrm{d}_{\mathbf{x y}}$ orbital is degenerate with $\mathrm{d}_{\mathrm{xz}}$ and $\mathrm{d}_{\mathrm{yz}}$ orbitals. ${ }^{f}$ The calculated density of states is only available for the bromide, which has not been synthesized experimentally. The isostructural $\mathrm{Cs}_{2} \mathrm{NaInCl}_{6} \mathrm{has}$ been synthesized. $g$ "MA" corresponds to $\mathrm{CH}_{3} \mathrm{NH}_{3}{ }^{+} .{ }^{h}$ The reported density of states does not distinguish between $\mathrm{Tl}^{+}$and $\mathrm{Tl}^{3+}$ contributions. These were determined based on chemical reasoning.

reasoning alone (considering the electronic configuration of the metals). We consider this a primary advantage of the LCAO approach as one can often rapidly obtain the most salient aspects of the band structure from nothing more than the chemical formula and Table 1. In more ambiguous cases, it is possible to use experimental orbital energies (determined from photoelectron spectroscopy) ${ }^{45}$ to identify the correct band composition. We used this approach in Section 3.2 to determine that the Sn $4 \mathrm{~d}$ orbitals are not involved in the $\mathrm{Cs}_{2} \mathrm{Sn}_{\square} \square \mathrm{Br}_{6}$ valence band. However, for many orbitals (particularly the metal LUMOs), photoelectron data are not available. To remedy this, we are currently compiling a database of frontier orbital energies for a variety of metal cations, which will be presented in a future publication.

We also must caution the reader against applying this LCAO theory too aggressively in compounds that lack dispersive bands. Flatter bands imply weaker $\mathrm{B}-\mathrm{X}$ and $\mathrm{X}-\mathrm{X}$ interactions either due to greater energetic mismatch between $\mathrm{B}$ and $\mathrm{X}$ orbitals or reduced $\mathrm{X}-\mathrm{X}$ overlap resulting from smaller $\mathrm{X}$ anions (e.g., $\mathrm{F}^{-}$replacing $\mathrm{I}^{-}$). To construct Table 1 , we have focused only on $\mathrm{B}-\mathrm{X}$ and the $90^{\circ} \mathrm{X}-\mathrm{X}$ interactions and neglected other effects (for example the $180^{\circ} \mathrm{X}-\mathrm{X}$ interactions). If the $\mathrm{B}-\mathrm{X}$ and $90^{\circ} \mathrm{X}-\mathrm{X}$ interactions are weak, then these neglected effects may indeed influence the band extrema. Additionally, flatter bands imply that the absolute energetic differences between different $\mathbf{k}$ points are small, making distinguishing between them more difficult and less meaningful.
It is also important to recognize that our theory is a nonrelativistic one and does not account for the effects of spin-orbit coupling (SOC) on the band structure. Despite this, we obtain remarkable correspondence between our predictions and DFT calculations, which include SOC, even in compounds with heavy elements where relativistic effects are known to be significant. In general, SOC can be viewed as a relatively small perturbation to the overall energy of the electronic states. The primary effects of this perturbation are additional splitting of nominally degenerate bands derived from $\mathrm{p}$ and $\mathrm{d}$ orbitals. The relative dispersion of each band is also affected to a lesser extent.

In all of the double perovskites examined here, SOC does not change the position of the VBM or CBM. Nevertheless, in Section 3.1, we noted two examples in which SOC leads to subtle variations in band structure, providing a reminder that it is important to consider potential effects of SOC when applying this theory.

\subsection{Extensions of the theory}

In this work, we have focused exclusively on double perovskites that adopt the undistorted cubic $F m \overline{3} m$ structure, accounting for most known halide double perovskite compositions. However, this excludes a number of double perovskites that show tilting between octahedra (for example, $\left.\mathrm{Rb}_{2} \mathrm{Sn}^{\mathrm{IV}} \square \mathrm{I}_{6}\right)^{51}$ or contain metals that exhibit Jahn-Teller distortions, which lower the local symmetry from octahedral point symmetry (e.g., 
$\left.\mathrm{Cs}_{2} \mathrm{Au}^{\mathrm{I}} \mathrm{Au}^{\mathrm{III}} \mathrm{Cl}_{6}\right) .^{52}$ The theory can be adapted to these cases as well, and we will provide a detailed analysis in future work.

We also see opportunities in extending the LCAO theory to address more complicated situations. In particular, there have been many recent reports of complex, non-stochiometric mixtures of B-site cations ${ }^{16,21,39,46,49,53}$ which, due to their disorder and low concentrations of dopants, are difficult to model computationally. We expect that our LCAO theory can accurately describe several limiting cases in these alloyed systems, potentially allowing for rapid identification of interesting and useful double perovskite alloys.

\section{Conclusions}

We demonstrate a qualitative approach for determining band structures of double perovskites with a high level of accuracy. Beginning from the double perovskite crystal structure and using basic LCAO methodologies, we explicitly determine all possible halide wavefunctions at the high-symmetry $\mathbf{k}$ points $\boldsymbol{\Gamma}$, $\mathbf{X}$, and $\mathbf{L}$. By examining how these wavefunctions interact with the B-site metal frontier orbitals as well as the halide-halide interactions within the wavefunctions themselves, we are able to accurately predict the band extrema for almost all experimentally known halide double perovskites. In many cases, the direct/indirect nature of the bandgap of a halide double perovskite can be estimated immediately from the material's atomic composition. Our results show that double perovskite band structures are dominated by the match or mismatch of symmetry between the frontier orbitals of the B- and $\mathrm{B}^{\prime}$-site metals. We also highlight the important role that halide-halide interactions, specifically those between halides oriented $90^{\circ}$ from one another, have in determining band dispersion when there is no contribution to the band from a B-site orbital. Importantly, we find that when the $\mathrm{B}$ and $\mathrm{B}^{\prime}$ cations are isoelectronic and participate in the band edges, a direct bandgap at $\boldsymbol{\Gamma}$ is expected, whereas other cases can produce indirect or direct bandgaps (Tables 1 and 2). We hope that this theory will provide a platform for developing an intuitive understanding of double perovskite band structures and that Table 1 serves as a useful guide for identifying new and interesting double perovskite compositions.

\section{Conflicts of interest}

There are no conflicts to declare.

\section{Note added after first publication}

This article replaces the version published on 30 Sep 2019, which contained an error in the first equation in section 2.3.

\section{Acknowledgements}

This work was supported by the Department of Energy, Office of Basic Energy Sciences, Division of Materials Sciences and Engineering, under contract DE-AC02-76SF00515. B. A. C. is supported by an NSF graduate fellowship (DGE-114747). L. L. acknowledges support by the Bavarian State Ministry of Science, Research, and the Arts through the grant "Solar Technologies go Hybrid (SolTech)", the Elite Network Bavaria, and computational resources provided by the Bavarian Polymer Institute and the German Research Foundation (DFG) through SFB840.

\section{Notes and references}

1 J. G. Bednorz and K. A. Müller, Z. Phys. B: Condens. Matter, 1986, 64, 189.

2 S. Royer, D. Duprez, F. Can, X. Courtois, C. Batiot-Dupeyrat, S. Laassiri and H. Alamdari, Chem. Rev., 2014, 114, 10292.

3 C. M. Combes, P. Dorenbos, C. W. E. van Eijk, K. W. Krämer and H. U. Güdel, J. Lumin., 1999, 82, 299.

4 Y. Li, W. Zhou, S. Xin, S. Li, J. Zhu, X. Lü, Z. Cui, Q. Jia, J. Zhou, Y. Zhao and J. B. Goodenough, Angew. Chem., Int. Ed., 2016, 55, 9965.

5 M. Li, M. J. Pietrowski, R. A. De Souza, H. Zhang, I. M. Reaney, S. N. Cook, J. A. Kilner and D. C. Sinclair, Nat. Mater., 2014, 13, 31.

6 Y. Zhou, X. Guan, H. Zhou, K. Ramadoss, S. Adam, H. Liu, S. Lee, J. Shi, M. Tsuchiya, D. D. Fong and S. Ramanathan, Nature, 2016, 534, 231.

7 NREL record efficiency chart. <https:/www.nrel.gov/pv/ assets/pdfs/best-research-cell-efficiencies-190416.pdf $>$

Accessed June 12, 2019. This plot is courtesy of the National Renewable Energy Laboratory.

8 A. Kojima, K. Teshima, Y. Shirai and T. Miyasaka, J. Am. Chem. Soc., 2009, 131, 6050.

9 A. Babayigit, A. Ethirajan, M. Muller and B. Conings, Nat. Mater., 2016, 15, 247.

10 A. H. Slavney, R. W. Smaha, I. C. Smith, A. Jaffe, D. Umeyama and H. I. Karunadasa, Inorg. Chem., 2017, 56, 46.

11 A. H. Slavney, T. Hu, A. M. Lindenberg and H. I. Karunadasa, J. Am. Chem. Soc., 2016, 138, 2138.

12 E. T. McClure, M. R. Ball, W. Windl and P. M. Woodward, Chem. Mater., 2016, 28, 1348.

13 G. Volonakis, M. R. Filip, A. A. Haghighirad, N. Sakai, B. Wenger, H. J. Snaith and F. Giustino, J. Phys. Chem. Lett., 2016, 7, 1254.

14 C. W. Cross and W. F. Hillebrand, Am. J. Sci., 1883, 26, 271. 15 J. Huang, Y. Yuan, Y. Shao and Y. Yan, Nat. Rev. Mater., 2017, 2,17042 .

16 A. H. Slavney, L. Leppert, D. Bartesaghi, A. Gold-Parker, M. F. Toney, T. J. Savenije, J. B. Neaton and H. I. Karunadasa, J. Am. Chem. Soc., 2017, 139, 5015.

17 A. H. Slavney, L. Leppert, A. Saldivar Valdes, D. Bartesaghi, T. J. Savenije, J. B. Neaton and H. I. Karunadasa, Angew. Chem., Int. Ed., 2018, 57, 12765.

18 G. Volonakis, A. A. Haghighirad, R. L. Milot, W. H. Sio, M. R. Filip, B. Wenger, M. B. Johnston, L. M. Herz, H. J. Snaith and F. Giustino, J. Phys. Chem. Lett., 2017, 8, 772.

19 Z. Deng, F. Wei, S. Sun, G. Kieslich, A. K. Cheetham and P. D. Bristowe, J. Mater. Chem. A, 2016, 4, 12025.

20 F. Wei, Z. Deng, S. Sun, F. Xie, G. Kieslich, D. M. Evans, M. A. Carpenter, P. D. Bristowe and A. K. Cheetham, Mater. Horiz., 2016, 3, 328. 
21 T. T. Tran, J. R. Panella, J. R. Chamorro, J. R. Morey and T. M. McQueen, Mater. Horiz., 2017, 4, 688.

22 M. R. Filip and F. Giustino, J. Phys. Chem. C, 2016, 120, 166.

23 Y. Cai, W. Xie, H. Ding, Y. Chen, K. Thirumal, L. H. Wong, N. Mathews, S. G. Mhaisalkar, M. Sherburne and M. Asta, Chem. Mater., 2017, 29, 7740.

24 X.-G. Zhao, D. Yang, Y. Sun, T. Li, L. Zhang, L. Yu and A. Zunger, J. Am. Chem. Soc., 2017, 139, 6718.

25 S. Körbel, M. A. L. Marques and S. Botti, J. Mater. Chem. C, 2016, 4, 3157.

26 F. A. Faber, A. Lindmaa, O. A. von Lilienfeld and R. Armiento, Phys. Rev. Lett., 2016, 117, 135502.

27 F. Bloch, Z. Phys., 1929, 52, 555.

28 J. C. Slater and G. F. Koster, Phys. Rev., 1954, 94, 1498.

29 R. Hoffmann, Solids and Surfaces: A Chemist's View of Bonding in Extended Structures, Wiley-VCH, 1988.

30 M. G. Goesten and R. Hoffmann, J. Am. Chem. Soc., 2018, 140, 12996.

31 S. Boyer-Richard, C. Katan, B. Traoré, R. Scholz, J.-M. Jancu and J. Even, J. Phys. Chem. Lett., 2016, 7, 3833.

32 M. Kim, J. Im, A. J. Freeman, J. Ihm and H. Jin, Proc. Natl. Acad. Sci. U. S. A., 2014, 111, 6900.

33 T. Wolfram and S. Ellialtıŏlu, Electronic and Optical Properties of $d$-Band Perovskites, Cambridge University Press, 2006.

34 J. M. Honig, J. O. Dimmock and W. H. Kleiner, J. Chem. Phys., 1969, 50, 5232.

35 A. H. Kahn and A. J. Leyendecker, Phys. Rev., 1964, 135, A1321.

36 N. W. Ashcroft and N. D. Mermin, Solid State Physics, Holt, Rinehart, and Winston, 1976.

37 W. A. Harrison, Electronic Structure and the Properties of Solids, W. H. Freeman, 1980.

38 B. Lee, C. C. Stoumpos, N. Zhou, F. Hao, C. Malliakas, C.-Y. Yeh, T. J. Marks, M. G. Kanatzidis and R. P. H. Chang, J. Am. Chem. Soc., 2014, 136, 15379.
39 A. E. Maughan, A. M. Ganose, M. M. Bordelon, E. M. Miller, D. O. Scanlon and J. R. Neilson, J. Am. Chem. Soc., 2016, 138, 8453.

40 N. Sakai, A. A. Haghighirad, M. R. Filip, P. K. Nayak, S. Nayak, A. Ramadan, Z. Wang, F. Giustino and H. J. Snaith, J. Am. Chem. Soc., 2017, 139, 6030.

41 B. Kang and K. Biswas, J. Phys. Chem. C, 2016, 120, 12187.

42 M.-G. Ju, M. Chen, Y. Zhou, H. F. Garces, J. Dai, L. Ma, N. P. Padture and X. C. Zeng, ACS Energy Lett., 2018, 3, 297. 43 P. A. Grutsch, M. V. Zeller and T. P. Fehlner, Inorg. Chem., 1973, 12, 1431.

44 W. Meng, X. Wang, Z. Xiao, J. Wang, D. B. Mitzi and Y. Yan, J. Phys. Chem. Lett., 2017, 8, 2999.

45 NIST X-ray Photoelectron Spectroscopy Database, National Institute of Standards and Technology, Gaithersburg, MD, https://srdata.nist.gov/xps/.

46 K. Du, W. Meng, X. Wang, Y. Yan and D. B. Mitzi, Angew. Chem., Int. Ed., 2017, 56, 8158.

47 M.-H. Du and K. Biswas, J. Lumin., 2013, 143, 710.

48 H. Shi and M.-H. Du, Phys. Rev. Appl., 2015, 3, 054005.

49 J. Luo, X. Wang, S. Li, J. Liu, Y. Guo, G. Niu, L. Yao, Y. Fu, L. Gao, Q. Dong, C. Zhao, M. Leng, F. Ma, W. Liang, L. Wang, S. Jin, J. Han, L. Zhang, J. Etheridge, J. Wang, Y. Yan, E. H. Sargent and J. Tang, Nature, 2018, 563, 541.

50 M. Retuerto, Z. Yin, T. J. Emge, P. W. Stephens, M.-R. Li, T. Sarkar, M. C. Croft, A. Ignatov, Z. Yuan, S. J. Zhang, C. Jin, R. Paria Sena, J. Hadermann, G. Kotliar and M. Greenblatt, Inorg. Chem., 2015, 54, 1066.

51 A. E. Maughan, A. M. Ganose, M. A. Almaker, D. O. Scanlon and J. R. Neilson, Chem. Mater., 2018, 30, 3909.

52 N. Elliott and L. Pauling, J. Am. Chem. Soc., 1938, 60, 1846.

53 K. P. Lindquist, S. A. Mack, A. H. Slavney, L. Leppert, A. GoldParker, J. F. Stebbins, A. Salleo, M. F. Toney, J. B. Neaton and H. I. Karunadasa, Chem. Sci., 2019, DOI: 10.1039/ C9SC02581B. 